\title{
Mechanistic evaluation of primary human hepatocyte culture using global proteomic analysis reveals a selective dedifferentiation profile
}

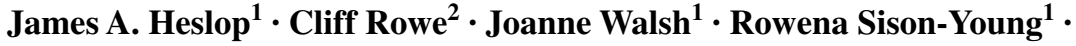 \\ Roz Jenkins ${ }^{1} \cdot$ Laleh Kamalian $^{1} \cdot$ Richard Kia $^{1} \cdot$ David Hay $^{1} \cdot$ Robert P. Jones $^{3}$. \\ Hassan Z. Malik ${ }^{3} \cdot$ Stephen Fenwick $^{3} \cdot$ Amy E. Chadwick $^{1} \cdot$ John Mills $^{4}$. \\ Neil R. Kitteringham ${ }^{1}$ - Chris E. P. Goldring ${ }^{1}$ B. Kevin Park ${ }^{1}$
}

Received: 17 July 2015 / Accepted: 21 March 2016 / Published online: 2 April 2016

(C) The Author(s) 2016. This article is published with open access at Springerlink.com

\begin{abstract}
The application of primary human hepatocytes following isolation from human tissue is well accepted to be compromised by the process of dedifferentiation. This phenomenon reduces many unique hepatocyte functions, limiting their use in drug disposition and toxicity assessment. The aetiology of dedifferentiation has not been well defined, and further understanding of the process would allow the development of novel strategies for sustaining the hepatocyte phenotype in culture or for improving protocols for maturation of hepatocytes generated from stem cells. We have therefore carried out the first proteomic comparison of primary human hepatocyte differentiation. Cells were cultured for $0,24,72$ and $168 \mathrm{~h}$ as a monolayer in order to permit unrestricted hepatocyte dedifferentiation, so as to reveal the causative signalling pathways and factors
\end{abstract}

Electronic supplementary material The online version of this article (doi:10.1007/s00204-016-1694-y) contains supplementary material, which is available to authorized users.

Chris E. P. Goldring

Chrissy@liv.ac.uk

James A. Heslop

jheslop@liv.ac.uk

1 Division of Molecular and Clinical Pharmacology, The Institute of Translational Medicine, MRC Centre for Drug Safety Science, The University of Liverpool, Liverpool L69 3GE, UK

2 CN Bio, Centre for Innovation and Enterprise, Oxford University Begbroke Science Park, Begbroke, Oxfordshire OX5 1PF, UK

3 University Hospital Aintree, Longmoor Lane, Liverpool L9 7AL, UK

4 AstraZeneca, Personalised Healthcare and Biomarkers, Alderley Park, Cheshire SK10 4TG, UK in this process, by pathway analysis. A total of 3430 proteins were identified with a false detection rate of $<1 \%$, of which 1117 were quantified at every time point. Increasing numbers of significantly differentially expressed proteins compared with the freshly isolated cells were observed at $24 \mathrm{~h}$ (40 proteins), $72 \mathrm{~h}$ (118 proteins) and $168 \mathrm{~h} \mathrm{(272}$ proteins) $(p<0.05)$. In particular, cytochromes $\mathrm{P} 450$ and mitochondrial proteins underwent major changes, confirmed by functional studies and investigated by pathway analysis. We report the key factors and pathways which underlie the loss of hepatic phenotype in vitro, particularly those driving the large-scale and selective remodelling of the mitochondrial and metabolic proteomes. In summary, these findings expand the current understanding of dedifferentiation should facilitate further development of simple and complex hepatic culture systems.

Keywords Cytochrome P450s - Human hepatocytes . Mass spectrometry $\cdot$ Mitochondria $\cdot$ iTRAQ $\cdot$ Donorvariation

$\begin{array}{ll}\text { Abbreviations } \\ \text { PHH } & \text { Primary human hepatocytes } \\ \text { DILI } & \text { Drug-induced liver injury } \\ \text { CYP } & \text { Cytochrome P450 } \\ \text { LETFs } & \text { Liver-enriched transcription factors } \\ \text { HNF } & \text { Hepatic nuclear factor } \\ \text { HLC } & \text { Hepatocyte-like cell } \\ \text { MAPKs } & \text { Mitogen-activated protein kinases } \\ \text { NFKB } & \text { Nuclear factor kappa-light-chain-enhancer of } \\ & \text { activated B cells } \\ \text { DEPs } & \text { Differentially expressed proteins } \\ \text { ROS } & \text { Reactive oxygen species } \\ \text { HSF } & \text { Heat shock factor protein } \\ \text { ERK } & \text { Extracellular signal-regulated kinase }\end{array}$


BSEP Bile salt export pump

MDR Multiple drug resistance protein

MRP Multidrug resistance-related protein

SLC Solute carrier family of transporters

\section{Introduction}

Primary human hepatocytes (PHHs) are an important tool for studying human liver disease and drug-induced liver injury (DILI), with other models, such as rat hepatocytes, hepatic cell lines and stem cell-derived hepatocyte-like cells failing to offer metabolically competent and speciesrelevant alternatives (Godoy et al. 2013). However, accessing and culturing PHHs remains difficult due to inconsistent supply and an inability to stimulate sufficient division for in vitro expansion. Furthermore, following isolation, primary human hepatocytes undergo a rapid dedifferentiation process in which many unique hepatocyte characteristics are lost, reducing the physiological relevance of the model (Fraczek et al. 2013; Godoy et al. 2013).

It is thought that dedifferentiation is caused by inflammatory and proliferative-associated responses to both PHH isolation and subsequent in vitro culture (Elaut et al. 2006; Fraczek et al. 2013; Godoy et al. 2009; Zellmer et al. 2010). Current models of dedifferentiation hypothesise that these responses result in the down-regulation of important liver-enriched transcription factors (LETFs) such as hepatic nuclear factors (HNFs), HNF1 $\alpha$ and HNF4 $\alpha$, which control many of the ADME processes (absorption, distribution, metabolism and excretion) and other features unique to PHHs, resulting in a loss of hepatic phenotype (Godoy et al. 2009; Zellmer et al. 2010); however, the exact mechanisms which govern these changes remain incompletely understood.

Several approaches have been adopted to maintain the hepatocyte phenotype in culture, including co-culture and 3D models such as sandwich cultures, bioreactors, liver buds and spheroids which attempt to better replicate the in vivo hepatic environment (Darnell et al. 2012; No et al. 2012; Tostoes et al. 2012). In addition, a wide range of growth factors, cytokines and small molecules have also been used to target and maintain the hepatic phenotype, such as those shown recently by Shan et al. (Shan et al. 2013). Many of these strategies have been thoroughly reviewed by both Godoy et al. (2013) and Fraczek et al. (2013).

Despite these continued improvements and advances towards complex culture conditions which can maintain an improved phenotype over a period of weeks, the full in vivo phenotype has not yet been achieved. Therefore, there remains a real need to improve the understanding of the underlying causes of this process and to identify novel strategies/targets for intervention. Moreover, as complex models reduce the effects of dedifferentiation, analysis of these systems may result in the underlying causes being difficult to fully ascertain. Therefore, studies of more rudimentary models which result in more pronounced dedifferentiation may consequently provide a greater insight into the partially understood and currently unknown causes of dedifferentiation.

Crucially, increased understanding of dedifferentiation is also likely to be useful in guiding attempts to direct and maintain a hepatic phenotype from pluripotent cells in vitro. Current efforts worldwide have not taken a cell beyond a relatively immature hepatocyte (Kia et al. 2013), and it can be hypothesised that an integral part of this challenge is how we can capture the hepatic phenotype and prevent its loss.

Global studies offer an insight into how cells react in a holistic sense to a particular condition, and whilst efforts have been made to map changes in the hepatocyte transcriptome during dedifferentiation (Kim et al. 2010; Lasher et al. 2011), many changes key to the mature phenotype of the hepatocyte are not properly represented in such analysis. This is exemplified by the recent results, showing that only $\sim 40 \%$ of the variation in cell protein levels can be explained by differences in the corresponding mRNA levels (Schwanhausser et al. 2011). We have previously shown that the use of a global proteomic approach to phenotype rat hepatocytes can provide novel information on dedifferentiation (Rowe et al. 2010) and here look to build on that study using human cells.

We have therefore now followed PHHs over the first 7 days of monolayer culture, chosen specifically to exacerbate the effects of dedifferentiation. Using iTRAQ-based stable isotope labelling and pathway analysis, we have identified the key changes in the PHH proteome during dedifferentiation and the inter-donor variability, which exists not only in donor phenotype, but also in the subsequent dedifferentiation profile.

\section{Materials and methods}

\section{Primary human hepatocyte isolation}

Primary human hepatocytes were isolated using a previously described 2-step collagenase method (LeCluyse et al. 2005). Briefly, liver resections were received as surgical waste (Aintree hospital, Liverpool, UK) with full patient consent and ethical approval. The resections were perfused with HEPES-buffered saline (HBS; $10 \mathrm{mM}$ Hepes, $5 \mathrm{mM}$ $\mathrm{KCl}, 136 \mathrm{mM} \mathrm{NaCl}, 5 \mathrm{~g} / \mathrm{l}$ glucose), followed by digestion with Collagenase A or IV (Roche, Basel, Switzerland or Sigma-Aldrich, St. Louis, MO) in HBS containing $700 \mu \mathrm{M}$ $\mathrm{CaCl}_{2}$. The capsule was then opened and the digested cells separated using gauze. The suspension was centrifuged twice at $80 \mathrm{xg}$ for $5 \mathrm{~min}$ at $4{ }^{\circ} \mathrm{C}$ and resuspended in Williams $\mathrm{E}$ 
medium (Sigma-Aldrich, St. Louis, MO). Cells were plated onto collagen-I-coated plates (BD Dickinson, San Jose, CA) at $2.5 \times 10^{5}$ cells $/ \mathrm{cm}^{2}$ to achieve full confluency, in Williams E supplemented with $1 \%$ insulin-transferrin-selenium (Life Technologies, Carlsbad, CA), 2 mM L-glutamine (SigmaAldrich, St. Louis, MO), $10^{-7} \mathrm{M}$ dexamethasone and penicillin (100 units $/ \mathrm{ml}) / \mathrm{streptomycin}(100 \mu \mathrm{g} / \mathrm{ml})$ (SigmaAldrich, St. Louis, MO). After 3 h, non-attached cells were washed away and the culture medium was replaced.

\section{ITRAQ}

Fresh samples were collected directly after isolation, before cells were plated. Samples were spun at $80 \times g$ for $5 \mathrm{~min}$ and lysed in $100 \mu \mathrm{l}$ iTRAQ buffer. In total, 24, 72 and $168 \mathrm{~h}$ time points were collected directly from 6 wells of a 24-well plates in a total of $100 \mu \mathrm{l}$ iTRAQ buffer. Protein concentration was determined by Bradford assay. Protein lysates derived from five donors were labelled according to the manufacturer's instructions (Applied Biosystems, Foster City, CA). One hundred micrograms protein in $20 \mu \mathrm{l}$ of iTRAQ buffer was denatured, and the protein cysteine residues were reduced with tris(2-carboxyethyl)phosphine for $1 \mathrm{~h}$ at $60{ }^{\circ} \mathrm{C}$ and subsequently capped with methylmethanethiosulfate, before overnight digestion with reconstituted trypsin at $37^{\circ} \mathrm{C}$. Isopropanol was then added to each sample, before labelling with differentially weighted isobaric tags for $2 \mathrm{~h}$, at room temperature. The labelled samples were then pooled and made up to $5 \mathrm{~mL}$ with $10 \mathrm{mM}$ potassium dihydrogen phosphate $/ 25 \% \mathrm{w} / \mathrm{v}$ acetonitrile. The $\mathrm{pH}$ was then adjusted using concentrated phosphoric acid to $<\mathrm{pH} \mathrm{3}$, before cation-exchange chromatography, followed by identification with mass spectrometry, as described previously (Rowe et al. 2010, 2013). Samples were run across three 8-plex iTRAQ runs (table S1), and results obtained relative to each donor's fresh sample to control for inter-donor variation.

\section{Proteomic data analysis}

Following iTRAQ analysis, only proteins, which were present in all samples, identified with $95 \%$ confidence ( 2 or more peptides) or $99 \%$ confidence (single peptide) with a false detection rate (FDR) of less than $1 \%$ were statistically analysed using R open-source software (http://www.rproject.org/). The iTRAQ output was analysed and differentially expressed proteins (DEPs) identified using the linear models for microarray data (LIMMA) and $t$ test (multtest) modules as described previously (Ritchie et al. 2015; Rowe et al. 2010). The R script and raw iTRAQ outputs used to generate the data are provided as supplementary information. Statistical outputs ( $p$ value, Benjamini-Hochberg and log fold change) of these modules were presented as volcano plots, and proteins detected in all samples were subjected to hierarchical clustering and heatmap analysis.

\section{Further proteomic data analysis}

Individual trend analysis of CYPs and transporters detected in $\geq 3$ donors was assessed by one-way ANOVA. Coefficient of variance $(\mathrm{CV})$ was calculated as (standard deviation/mean). The most variable proteins were defined as $\mathrm{CV}>1.3$, and the most stable proteins were defined as $\mathrm{CV}<0.3$ and a mean relative fold change $>0.8$ and $<1.25$. PANTHER analysis was used to categorise differential subsets of proteins into biological functional groups and displayed as a pie chart (Mi et al. 2013).

\section{Pathway analysis}

Significant DEPs $(p<0.05)$ were uploaded to the Ingenuity platform IPA (Qiagen, Venlo, Netherlands) to investigate the associated pathways, networks and regulators. IPA uses a database of known protein interactions from scientific publications to associate and group the uploaded DEPs with pathways (Thomas and Bonchev 2010). Using IPA algorithms, the software generates a $p$ value which relates to the likelihood that a particular pathway or network is linked to the DEPs in the dataset. Only pathways that were altered by $p<0.05$ (Fisher exact $t$ test) were classed as significantly altered or linked. The Z-activation score, which additionally takes into account the directional change of the proteins, was used for biological function and upstream regulator analysis. Using the IPA algorithm, functions or regulators that have a Z-score of $\geq 2$ are predicted to be activated and $\leq-2$ are predicted to be inhibited.

\section{Transcription factor binding analysis}

Mapper $_{2}$ online software was used to compare the predicted transcription factor binding sites (Marinescu et al. 2005). Analysis was completed using the collated database, analysing the sequence of each gene 2000 base pairs upstream of the transcription start site. The number of proteins of interest which interacted with each predicted transcription factor was then compared to determine the significance of each factor. Those factors which demonstrated enriched predicted binding within a subset of proteins ( $\geq 4$ proteins) were classed of factors of interest.

\section{Western blotting}

Samples collected in iTRAQ buffer were quantified by Bradford assay and assessed by western blot to validate iTRAQ results. Briefly, $5 \mu \mathrm{g}$ samples were denatured at $80{ }^{\circ} \mathrm{C}$ in Laemmli sample buffer (Sigma-Aldrich, St. Louis, 
MO) and separated in $10 \%$ polyacrylamide gels and then transferred to nitrocellulose membranes (G.E Healthcare, Buckinghamshire, UK). Following $1 \mathrm{~h}$ blocking in $10 \%$ milk (Bio-Rad, Hercules, CA), primary antibodies directed against CYP2E1 (Abcam, Cambridge, UK; 1:5000), CYP2D6 (BD Gentest, San Jose, CA; 1:1000), CYP1A2 (Abcam, Cambridge, UK; 1:3000) were added overnight or for 15 min Actin (Abcam, Cambridge, UK; 1:10,000). Following washing, secondary mouse (1:10,000; CYP2D6, CYP1A2, Actin) or rabbit (1:5000; CYP2E1) antibodies were subsequently added for $1 \mathrm{~h}$. Membranes were then washed and visualised using chemiluminescence.

\section{Metabolism studies}

To determine the metabolic changes that occur in PHH during dedifferentiation, we used quantitative mass-spectrometry-based activity assays. PHHs were incubated for $15 \mathrm{~min}$ at $37{ }^{\circ} \mathrm{C}\left(5 \% \mathrm{CO}_{2}\right)$ at a final substrate cocktail concentration of $1 \mathrm{mM}$ testosterone (CYP3A4) and $0.25 \mathrm{mM}$ dextromethorphan (CYP2D6) (both purchased from Sigma-Aldrich, St. Louis, MO) in $\mathrm{MeOH}$ or $\mathrm{H}_{2} \mathrm{O}$, respectively (Fisher Scientific, Pittsburgh, PA), in culture media. $0.5 \mathrm{mM}$ Phenacetin (Sigma-Aldrich, St. Louis, MO) in $100 \% \mathrm{MeOH}$ was then added to the incubation media $(1: 1 \mathrm{v} / \mathrm{v})$ as a stop solution and an internal standard for LC-MS-MS analysis. The media containing the respective metabolites, $6 \beta-\mathrm{OH}$-testosterone and dextrorphan, were then quantified by LC-MS-MS. This was repeated for the $24,48,72$ and $168 \mathrm{~h}$ time points.

\section{Seahorse functional mitochondrial assay}

PHH isolated from subsequent donors were cultured on collagen-I-coated (Life Technologies, Carlsbad, CA; $50 \mu \mathrm{g} / \mathrm{ml}$ in $0.02 \mathrm{M}$ acetic acid), XF 96-well cell culture microplates (Seahorse biosciences, North Billerica, MA; $2.5 \times 10^{4}$ cells/well). OXPHOS stress test medium was supplemented with $25 \mathrm{mM}$ D-glucose, $2 \mathrm{mM}$ L-glutamine and $1 \mathrm{mM}$ sodium pyruvate (final concentration). The glycolytic stress test medium was prepared by adding $2 \mathrm{mM}$ L-glutamine (final concentration). Both media were pre-warmed to $37{ }^{\circ} \mathrm{C}$. PHH culture medium was removed and replaced with OXPHOS or glycolytic stress test medium. Cells were incubated in a $\mathrm{CO}_{2}$-free incubator at $37{ }^{\circ} \mathrm{C}$ for $1 \mathrm{~h} .1 \mu \mathrm{M}$ oligomycin, $0.25 \mu \mathrm{M}$ FCCP and $1 \mu \mathrm{M}$ rotenone-antimycin-A (OXPHOS stress test) and $25 \mathrm{mM}$ glucose, $1 \mu \mathrm{M}$ oligomycin, and $100 \mathrm{mM}$ 2-deoxyglucose solutions (glycolytic stress test) were prepared. Prior to the rate measurements, the XFe96 Instrument (Seahorse biosciences, North Billerica, MA) allowed the oxygen partial pressure to reach equilibrium. The oxygen consumption rate $(\mathrm{OCR})$ and extracellular acidification rate (ECAR) were measured simultaneously three times to establish a baseline rate. After each compound injection, conditions were allowed to return to normal oxygen tension and $\mathrm{pH}$. The OCR and ECAR measurements for each well were recorded and reported as $\mathrm{pmol} / \mathrm{min}$ and $\mathrm{mpH} / \mathrm{min}$, respectively, by XF Wave software. Results were displayed as a percentage of maximal OCR and ECAR or as relative fold change of each parameter between time points.

\section{Rotenone assay}

PHH was plated at $1 \times 10^{5}$ in 96-well collagen-I-coated plates. At 24 and $168 \mathrm{~h}$, serial concentrations $(0-20 \mathrm{mM})$ of rotenone (Sigma-Aldrich, St. Louis, MO) were made in DMSO (Fisher Scientific). Then, the compound solutions were added to culture media at $1 / 200(\mathrm{v} / \mathrm{v})$ ratio to make final dosing concentrations of $0-100 \mu \mathrm{M}(0.5 \% \mathrm{v} / \mathrm{v}$ DMSO). Culture media were removed and replaced with the media containing rotenone. Following incubation for $2 \mathrm{~h}\left(37^{\circ} \mathrm{C}, 5 \% \mathrm{CO}_{2}\right)$, ATP content was assessed using the CellTitre-Glo assay (Promega, Madison, WI), according to the manufacturers' instructions. Briefly, $30 \mu \mathrm{l}$ of ATP reagent was added to each well containing $100 \mu \mathrm{l}$ of media. The plate was shaken $(1 \mathrm{~min})$, and then, $100 \mu \mathrm{l}$ of the well content was transferred to a white 96-well plate, and the ATPase luminescence was measured using a plate reader (Varioskan, Thermo Scientific, Waltham, MA). Results were presented as percentage of control.

\section{Results}

\section{Global proteomics reveals donor-dependent variation as a major influence on dedifferentiation}

iTRAQ quantitative global proteomic analysis was used to assess the changes which occur in $\mathrm{PHH}$ at different time points during a 168-h monolayer culture. Dedifferentiation and loss of characteristic hepatocyte morphology were confirmed by microscopy. Figure 1a shows images of the cells taken at 24, 72 and $168 \mathrm{~h}$ and clearly demonstrates the maintenance of confluency and the loss of the typical hepatocyte polygonal morphology $(24 \mathrm{~h})$, towards a culture with flatter and less defined epithelial characteristics at 72 and $168 \mathrm{~h}$.

A total of 3430 unique proteins were detected across three iTRAQ runs (table S1), with 1117 identified across all samples. Statistical analysis using a linear model yielded significantly differentially expressed proteins (DEPs) at each time point relative to freshly isolated cells (table S2). These changes highlight a dynamic and escalating process; 40 DEPs are seen after $24 \mathrm{~h}, 118$ after $72 \mathrm{~h}$ and 272 after $168 \mathrm{~h}$ in culture $(p<0.05)$. When displayed as volcano plots (log fold change vs. $p$ value), the increasing dispersion and direction of change of the proteins over time in 
(a)
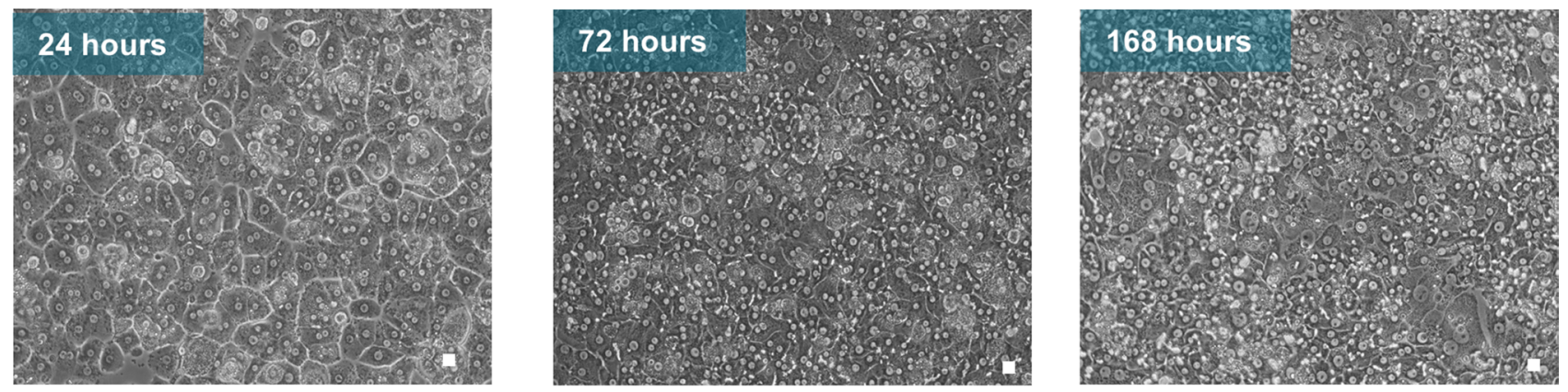

(b)

24 hours

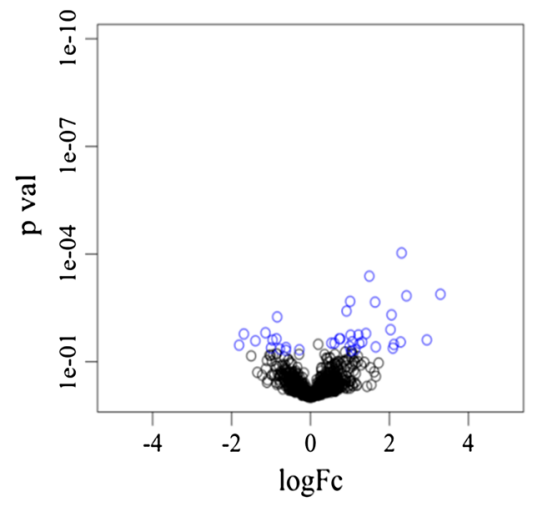

72 hours

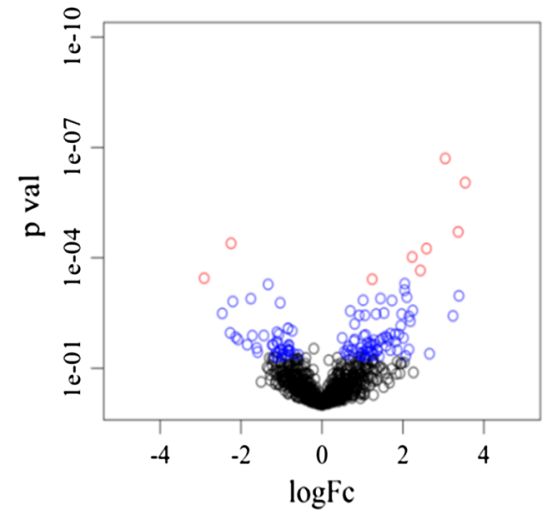

168 hours

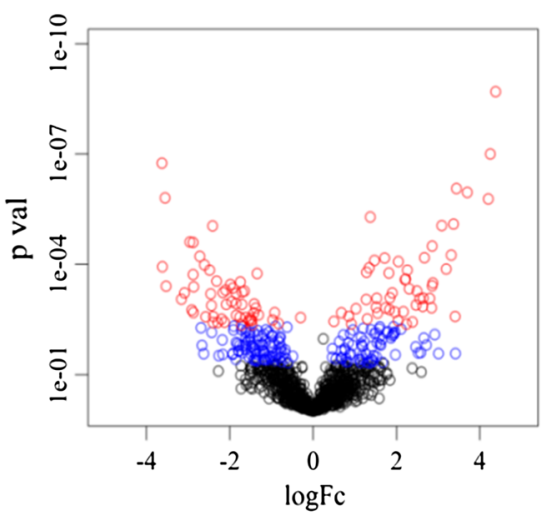

(c)
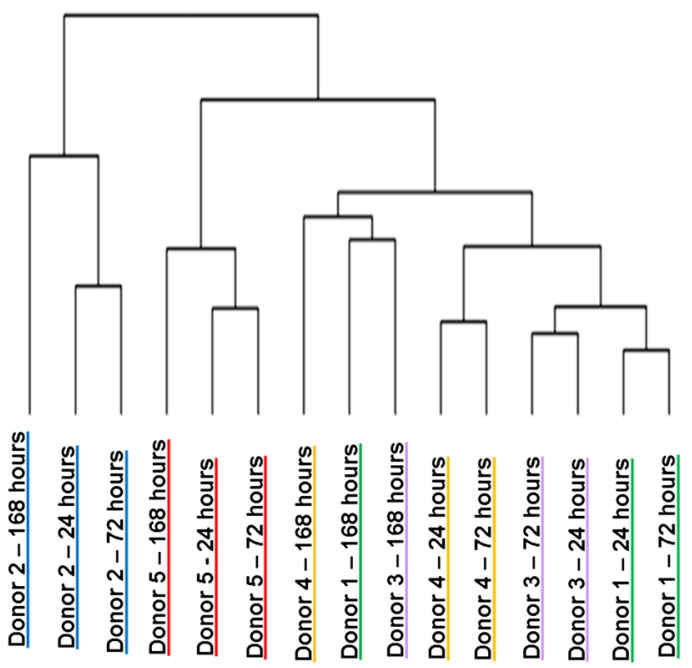

Fig. 1 Morphological and statistical analysis a morphological changes in primary human hepatocytes over $168 \mathrm{~h}$ in monolayer collagen-I-coated plate culture. Scale bar represents $10 \mu \mathrm{m}$; b volcano plot analysis of the iTRAQ detected proteins log fold change versus $p$ value at 24,72 and $168 \mathrm{~h}$. Blue significantly altered proteins $(n=5 ; p \leq 0.05)$ and red significantly altered after multiple testing (d)
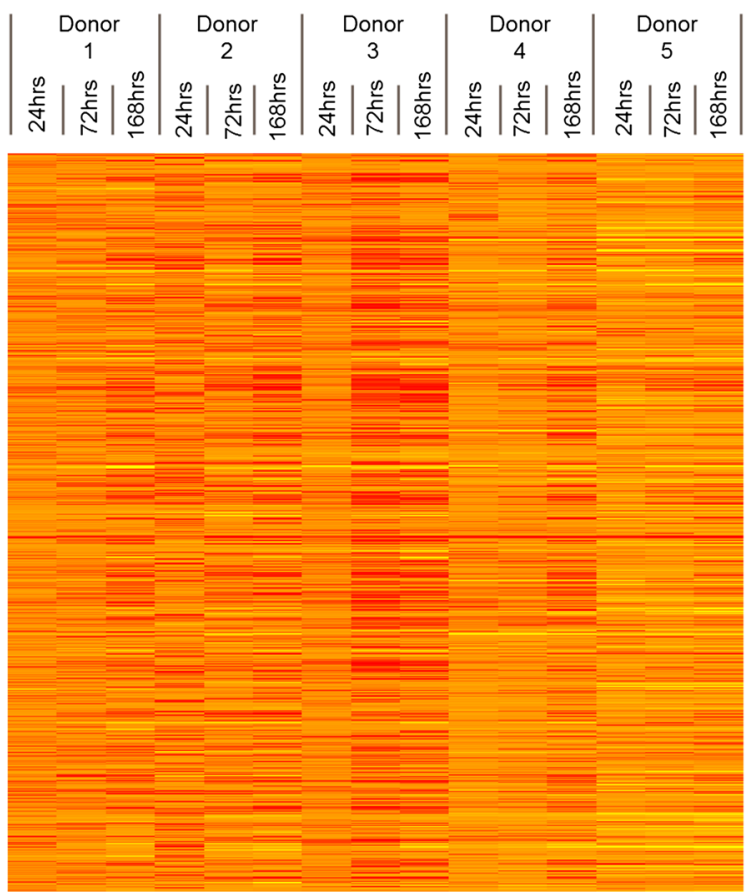

correction $(n=5$; Benjamini-Hochberg $\leq 0.05)$ c hierarchal clustering analysis showing the relationship between donors and time points $(n=5)$; $\mathbf{d}$ heatmap of each donor's proteome during dedifferentiation relative to the freshly isolated sample. Red indicates down-regulation, yellow up-regulation. The intensity of colour reflects the degree of change 
culture are strikingly evident (Fig. 1b); interestingly, at both 24 and $72 \mathrm{~h}$ of culture, the majority of DEPs increased in expression.

Hierarchical clustering of the entire dataset shows that the dominant factor in determining how the samples are related is in fact the original source of the cells, rather than time in culture (Fig. 1c). This is particularly pronounced at the 24 and $72 \mathrm{~h}$ time points, which cluster according to donor in every case. The separation from the other donors is still evident at $168 \mathrm{~h}$ for donors 2 and 5, although these samples do cluster away from the earlier time points, whereas by this time the other three donors (1, 3 and 4$)$ cluster together, demonstrating a convergence towards a dedifferentiated phenotype which is more reflective of time in culture than the cell source. Thus, these data suggest that phenotypic characteristics of the donor have a greater impact on the protein dedifferentiating expression profile of the hepatocytes than does the length of time in culture, particularly over the first $72 \mathrm{~h}$ of culture. The variability between donor proteomic profiles during dedifferentiation is further shown by the heatmap in Fig. 1d.

To identify the proteins with the greatest variability across the 5 donors at the different time points, coefficient of variance (CV) analysis was then used (table S3). This criterion highlights a number of proteins, including CYP2C9, which is known to vary in expression between individuals (Sistonen et al. 2009), and glutamine synthetase, which is associated with differential expression according to liver zonation (Jungermann and Kietzmann 1996). In fact, zonation appears to be a large influencing factor on variation during culture as our pathway analysis of the most variable proteins revealed significant association with ketogenesis, cholesterol biosynthesis, fatty acid $\beta$ oxidation and glutamine synthesis (table S4), all of these are related to the portocentral axis gradient of the lobule (Godoy et al. 2013).

The most stable proteins during dedifferentiation were also investigated (Table S5). These included several mitochondrial proteins within the ten most stable proteins. Thus, while many proteins show conserved differential expression or high levels of inter-donor variability, a subset of proteins remains relatively stable and consequently may retain in vivo-like functionality and also serve as useful normalisers for protein expression changes.

Table S6 highlights the most up- and down-regulated DEPs at each time point, as assessed by $\log _{2}$ fold change relative to the fresh sample. Interestingly, the first noticeable effects at $24 \mathrm{~h}$ are an increase in stress-related mediator superoxide dismutase and a decrease in histone proteins. Of note, hepatic-associated proteins are not universally down-regulated, as exemplified by alpha-1-antitrypsin and cytokeratins 8 and 18, which are up-regulated throughout the analysis (Table S2 and S6). Interestingly, these proteins
Fig. 2 Pathway analysis of dedifferentiation a biological functions associated with the DEPs according to Z-activation score. If score $2 \geq \mathrm{Z}$-score $\leq-2$, the function is predicted to be activated or inhibited, respectively. Red up-regulated, green down-regulated. Intensity of colour correlates with greater Z-activation score $\mathbf{b}$ canonical pathways during dedifferentiation. Results displayed-Log ( $p$ values), pathway included if $p<0.05$ at any of the assessed time points. Colour Intensity corresponds to the significance value; c upstream regulators predicted to be either activated or inhibited by Z-activation score $(2 \geq Z$-score $\leq-2)$. Red activated, green inhibited, in order of significance

are readily detectable in hepatocyte-like cells derived from stem cells (Rambhatla et al. 2003), whereas many of the down-regulated proteins are not, demonstrating that simple in vitro culture systems may support similar expression patterns during both hepatic dedifferentiation and differentiation.

\section{Pathway analysis reveals the key changes and dynamic profile of dedifferentiation}

Pathway enrichment analysis was used to identify the affected functions and pathways which underlie dedifferentiation. A list of the most significantly perturbed pathways and functions at the three time points is shown in table S7.

Heatmap analysis of the biological functions at the three post-isolation time points (Fig. 2a) shows the differential dynamics of the pathway groups. Many of the pathways follow a linear pattern of increasing activation or inhibition; however, some, such as the cell survival pathways, display different dynamics, as they are predicted to be activated at $72 \mathrm{~h}$, but not at 24 or $168 \mathrm{~h}$. Figure $2 \mathrm{~b}$ displays the canonical pathways at the assessed time points. Whilst metabolic pathways display a trend of increasing significance during culture, acute phase response signalling and ROS/NOS production are significantly affected at $24 \mathrm{~h}$, but not at $168 \mathrm{~h}$. Taken together, these data indicate a time point-dependent cellular response to isolation and culture (figure S1).

Upstream regulation analysis (i.e. transcription factors, cytokines, growth factors and receptors) was then used to predict which regulators were activated or inhibited (Fig. 2c). The results suggest an activation of proliferative and inflammatory regulators and inhibition of metabolicassociated factors. Moreover, many energy productionassociated factors are down-regulated at $168 \mathrm{~h}$ including $\operatorname{PPAR} \alpha$, insulin receptor, KLF15 and the mitochondrial transcription factor TFAM.

\section{Analysis of mitochondrial functional profile reveals donor variation in the bioenergetic profile}

Pathway analysis predicted a perturbation of energy production and mitochondrial mechanics; therefore, we investigated the mitochondrial proteome in further detail. The 
(a)

\section{Biological Functions}

Migration of cells

Cell movement

Cell death

Cell spreading

Necrosis

Hydroxylation of lipid

Organization of cytoplasm

Organization of cytoskeleton

Proliferation of cells

Cell viability

Oxidation of lipid

Cell survival

Oxidation of fatty acid

Fatty acid metabolism

Organization of organelle

Organismal death

Organization of filaments (b)

Canonical Pathway

Regulation of Cellular Mechanics by Calpain Protease Nicotine Degradation II

Fatty Acid Activation

Y-linolenate Biosynthesis II (Animals)

Mitochondrial L-carnitine Shuttle Pathway

Melatonin Degradation I

Nicotine Degradation III

FAK Signaling

4-aminobutyrate Degradation I

Glutamate Degradation III (via 4-aminobutyrate)

Actin Oytoskeleton Signaling

FXR/RXR Activation

Glucocorticoid Receptor Signaling

Superpathway of Melatonin Degradation

Stearate Biosynthesis I (Animals)

LPS/L-1 Mediated Inhibition of RXR Function

ILK Signaling

HIF1a Signaling

14-3-3-mediated Signaling

Fatty Acid $\beta$-oxidation I

NRF2-mediated Oxidative Stress Response

Paxillin Signaling

Bupropion Degradation

Acetone Degradation I (to Methylglyoxal)

LXR/RXR Activation

Aldosterone Signaling in Epithelial Cells

Role of Tissue Factor in Cancer

VEGF Signaling

Hypoxia Signaling in the Cardiovascular System

Estrogen Biosynthesis

Glycine Cleavage Complex

PPAR Signaling

Coagulation System

Integrin Signaling

Telomerase Signalin

NAD Biosynthesis III

5-aminoimidazole Ribonucleotide Biosynthesis I

Acute Phase Response Signaling

Production of Nitric Oxide and Reactive Oxygen Species

Clathrin-mediated Endocytosis Signaling

Inhibition of Matrix Metalloproteases
-Log (p-value)

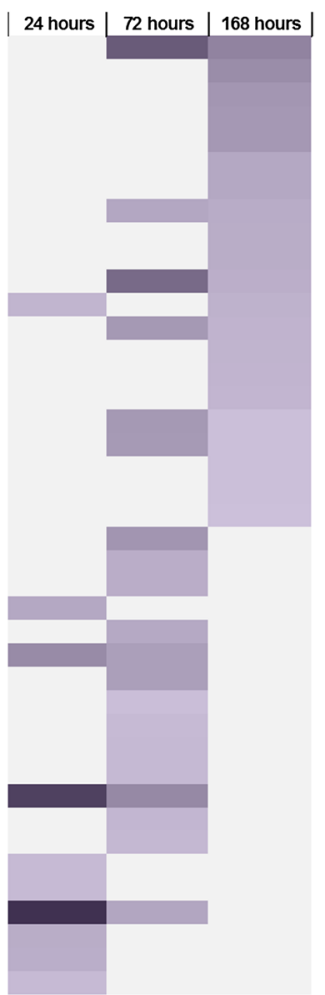

$-\log (p$ value $)$

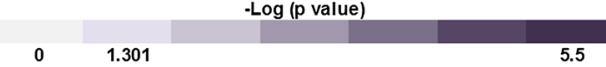

(c)

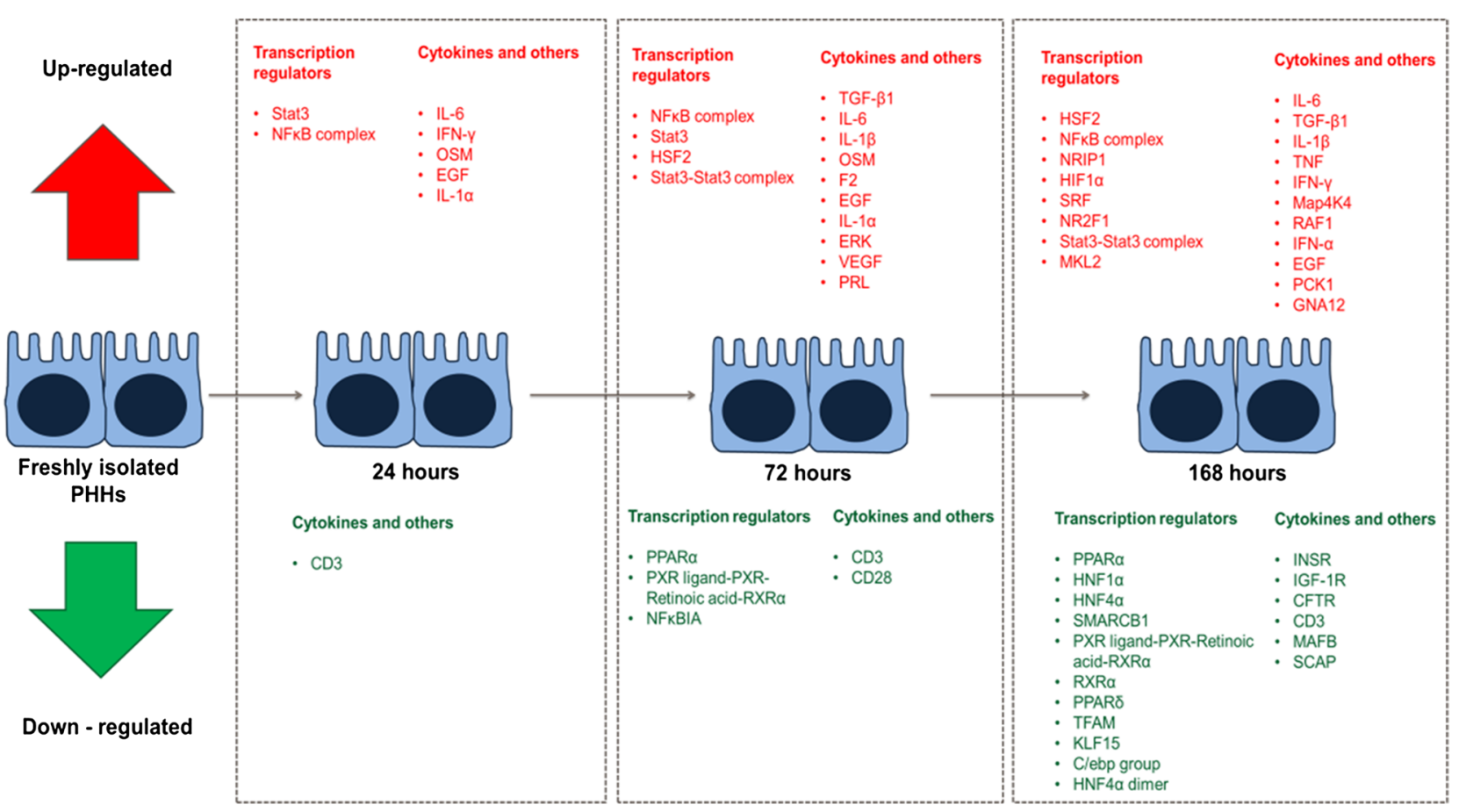


(a)

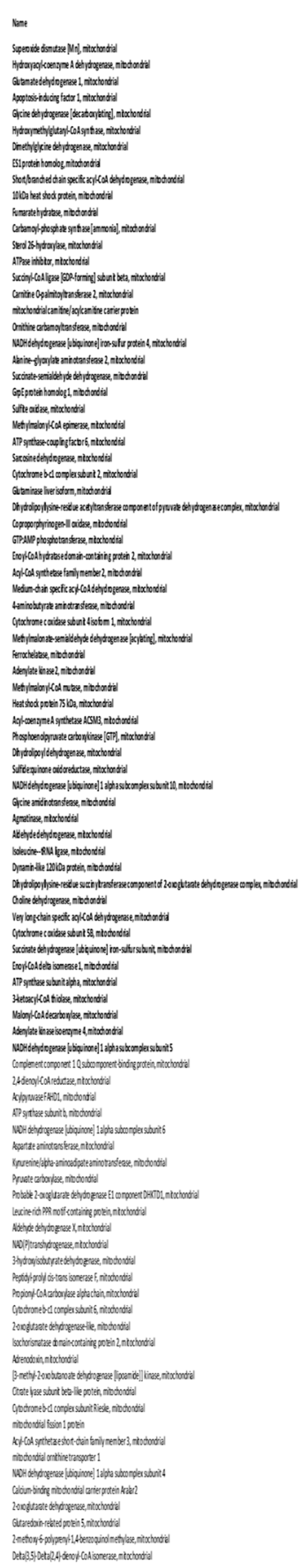

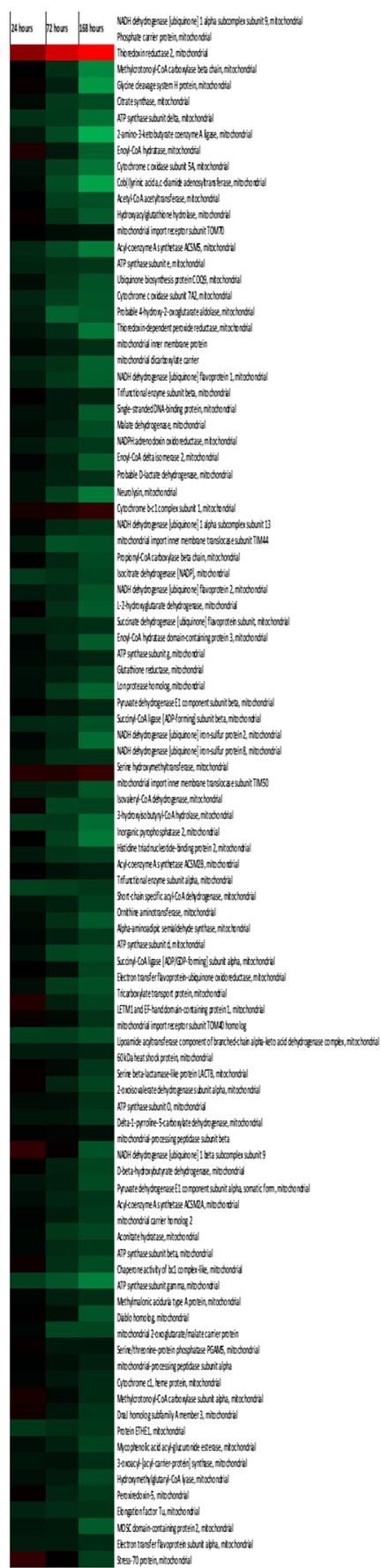

Log fold change (b)

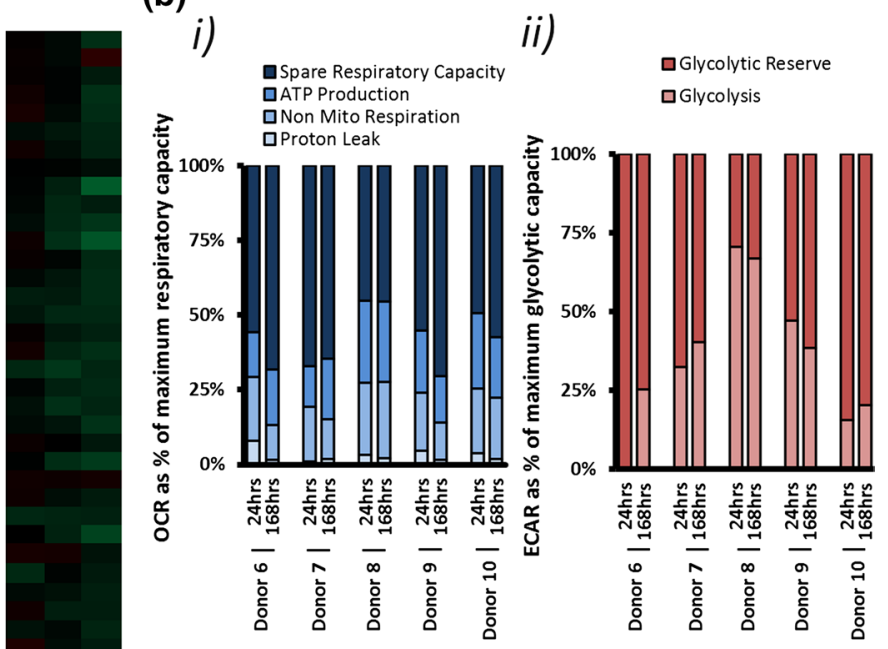

iii)
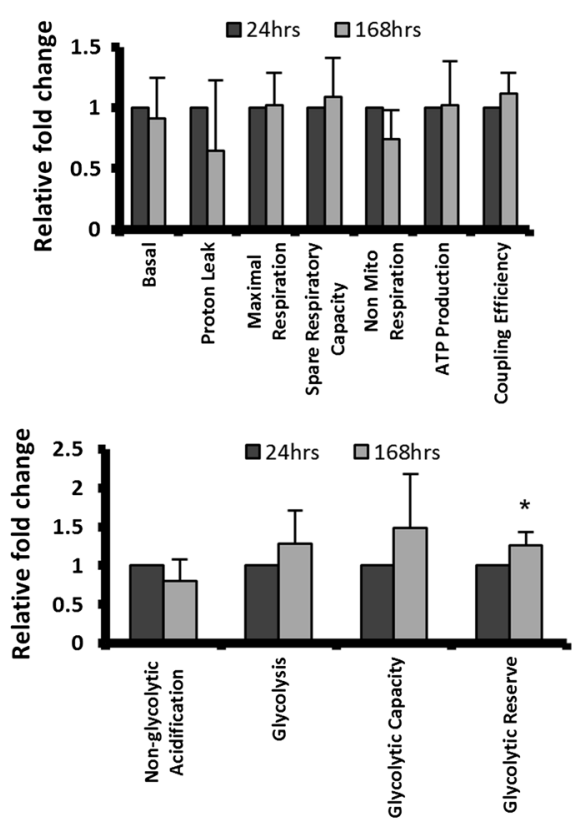

(c)

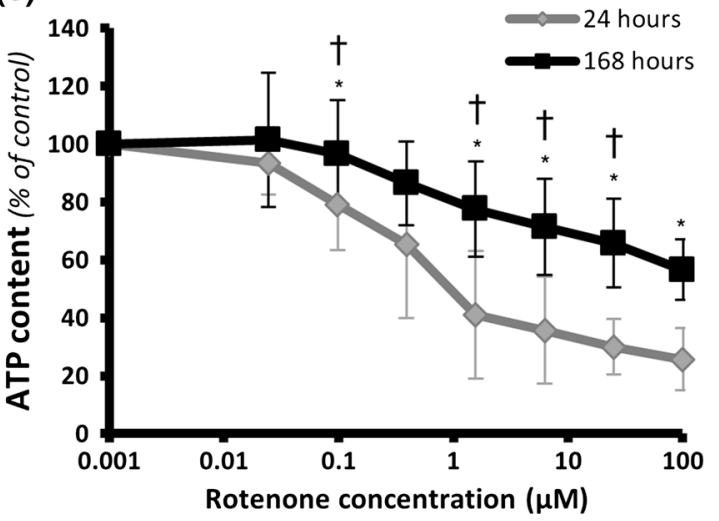


4Fig. 3 Mitochondrial changes during dedifferentiation. a $\log _{2}$ fold change of mitochondrial proteins. Red up-regulated, green down-regulated. Intensity of colour $\log _{2}$ fold change. Bold proteins differentially expressed at $168 \mathrm{~h}(p<0.05)$; b $(i)$ oxidative phosphorylation and (ii) glycolysis parameters as percentages of maximal oxygen consumption rates and extracellular acidification rate, respectively; relative fold change of (iii) oxidative phosphorylation and (iv) glycolysis parameters. $\left.{ }^{*}\right) p<0.05$ two-tailed paired t test; c ATP levels at 24 (filled diamond) and $168 \mathrm{~h}$ (filled square) following rotenone treatment. Results: percentage of control, $(*) p<0.05$ two-tailed paired $t$ test and $(\dagger) p<0.05$ Wilcoxon signed rank test $(n=5)$

expression of proteins with known mitochondrial associations demonstrated a general downward trend of the DEPs (bold) with time in culture, particularly at $168 \mathrm{~h}$, with superoxide dismutase II being a notable exception (Fig. 3a). However, a number of key mitochondrial proteins, such as mitochondrial import receptor subunits, remained largely unchanged (table S5). This indicates that the large-scale down-regulation of mitochondrial proteins represents an alteration in function between freshly isolated cells and $168 \mathrm{~h}$ rather than a generalised loss of mitochondrial mass between these time points.

Pathway analysis was then used to assess the mitochondrial proteomic dataset DEPs. This analysis revealed predicted roles for AMPK signalling, a master regulator of energy metabolism, which increases in significance during time in culture (Figure S2a). Furthermore, a negative regulator of mitochondrial fusion, OMA-1, is also predicted to be down-regulated (figure $\mathrm{S} 2 \mathrm{~b}$ ).

To assess how these changes impacted mitochondrial function, oxygen consumption rate (OCR) and extracellular acidification rate (ECAR) at 24 and $168 \mathrm{~h}$ were subsequently investigated in $\mathrm{PHH}$ isolated from a further five donors. The analysis demonstrated a clear variation in individual bioenergetic OCR profiles at $24 \mathrm{~h}$ (Fig. 3b (i)), with the 168-h profile showing greater dependence on the $24 \mathrm{~h}$ profile, rather than time in culture. Analysis of glycolytic consumption, as measured by ECAR, shows much greater donor-dependent variation (Fig. 3b (ii)), which may be attributable to the known gradient of glycolysis across the portocentral axis, and is consistent with our findings regarding the most variable proteins. Despite this variation, a trend emerges: basal glycolysis shows an increase during culture in donors with lower basal glycolysis, whereas those with higher basal levels at $24 \mathrm{~h}$ decrease, suggestive of a convergence towards a culture-dependent level of basal glycolysis.

Furthermore, many of the tested OCR parameters remain remarkably stable between 24 and 168 h (Fig. 3b (iii)), despite the large-scale changes to the mitochondrial proteome. ECAR measurements showed similar stability; however, there was a general trend of increased glycolytic parameters, including the only significantly altered factor, namely glycolytic reserve (Fig. 3b (iv)).
Further investigation of the proteomic data revealed a significant down-regulation of three complex I subunits, whereas the remaining nine detected subunits were not significantly differentially expressed. To assess the functional consequence of these changes, rotenone was used as a model of complex I mitochondrial perturbation. A significant increase in $\mathrm{PHH}$ resistance to rotenone toxicity in all donors at $168 \mathrm{~h}$ regardless of background bioenergetic (OCR/ECAR) profile (Fig. 3c) was found. Together these data suggest that, despite the variability seen between donors, a conserved change in essential mitochondrial function is observed as a result of time in culture.

\section{Assessment of ADME proteins during dedifferentiation reveals selective protein down-regulation}

A well-known feature of hepatocyte dedifferentiation is the loss of the metabolic phenotype. To distinguish whether this is a selective or global loss of metabolic competence, several protein subsets generated in this analysis were compared: 168 h DEPs, 168 h non-DEPs, most stable proteins and the most variable proteins. PANTHER analysis was used to compare the associated biological function categories of each subset (Figure S3). This analysis revealed similar percentages of proteins in each class, with the greatest proportion of proteins associated with metabolism in all subsets, indicating a selective, rather than wholesale alteration, in the hepatocyte metabolic profile.

Figure 4a shows the known phase I, II and III drugmetabolising enzymes (Guo et al. 2011), which were detected by proteomic analysis. The heatmap highlights the loss of metabolic competence with the majority of phase I enzymes following a relatively linear pattern of downregulation towards $168 \mathrm{~h}$. Phase II/III enzymes appear to show greater stability with some phase III proteins, including multidrug resistance protein 1 (MDR1) and major vault protein (MVP), demonstrating an up-regulation during culture.

Figure $4 \mathrm{~b}$ demonstrates the dedifferentiation pattern of the cytochrome P450 s detected by iTRAQ over the 168-h period. The general trend of expression relative to freshly isolated PHHs is downward; however, the majority of the detected CYP proteins are maintained in expression at $24 \mathrm{~h}$ in relation to the freshly isolated cells, showing that at $24 \mathrm{~h}$ the metabolic potential of $\mathrm{PHH}$ is mostly maintained (Fig. 4b and table S8). Some CYPs displayed greater stability, such as 2C18 and 4F11, whilst 2B6 and 2C9 demonstrated downward trends, but showed greater variation between the donors.

Western blotting for CYP proteins 2D6, 2E1 and 1A2 was consistent with the iTRAQ results, further demonstrating a downward trend with noted variation in the individual 
(a)
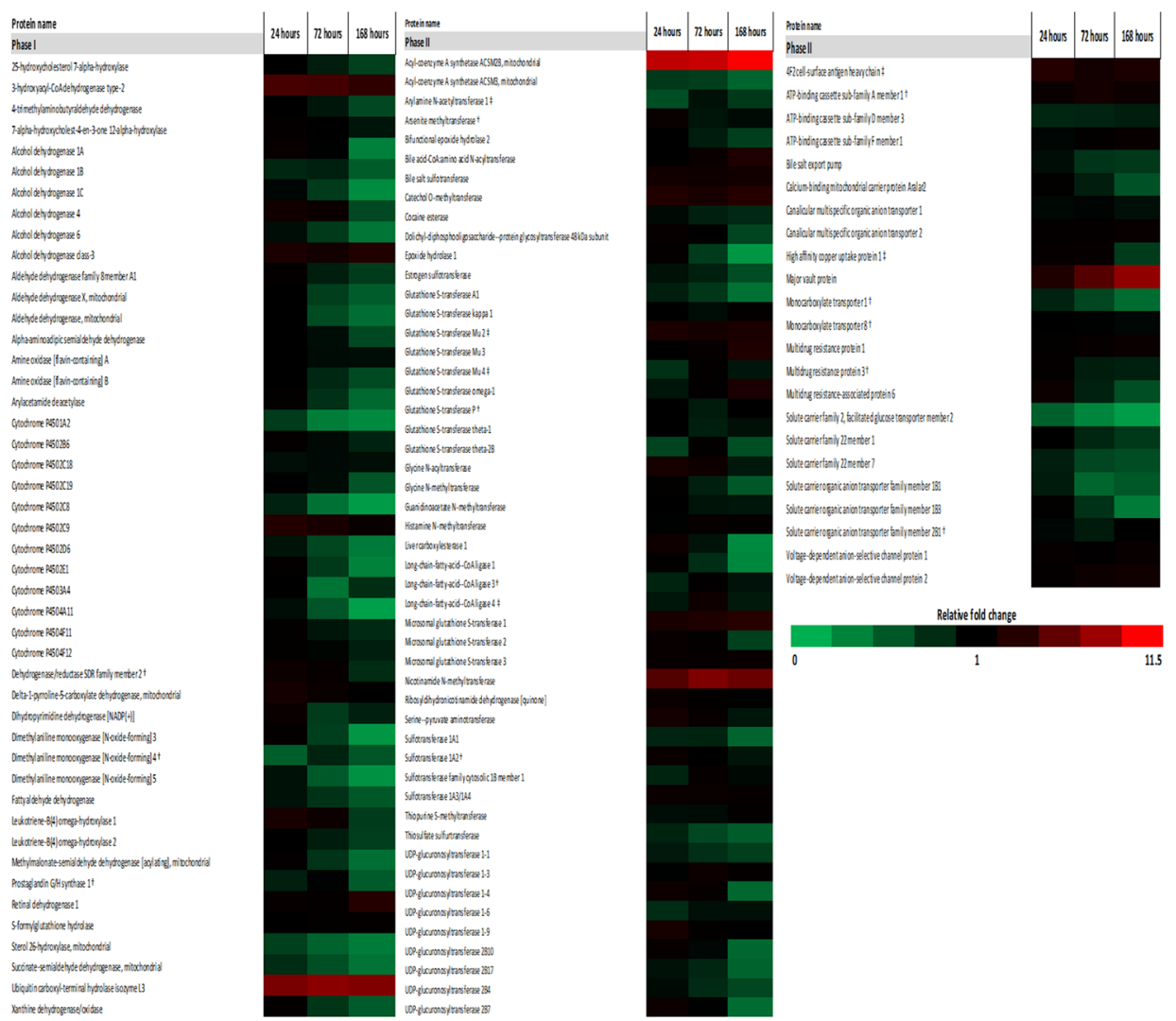

(b)

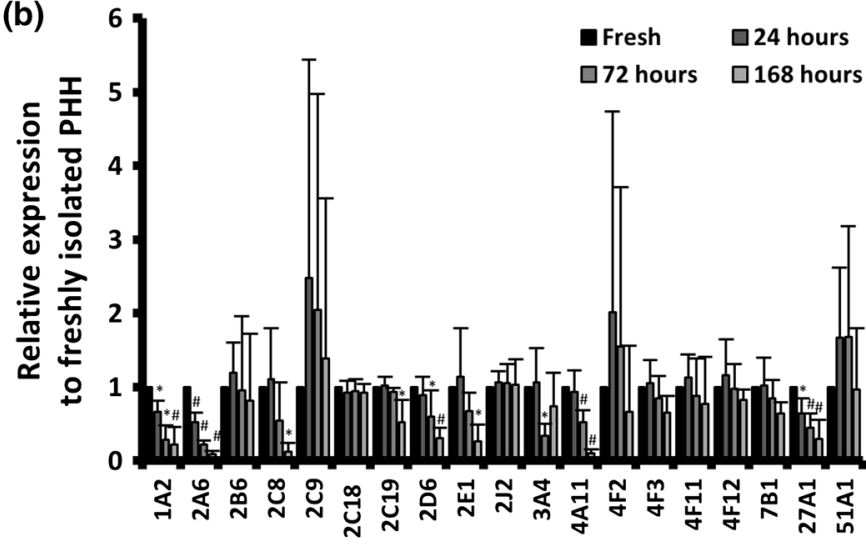

(c)

Fresh 24 hours 48 hours 72 hours 168 hours

CYP2E1

CYP2D6

CYP1A2

$\beta$-actin

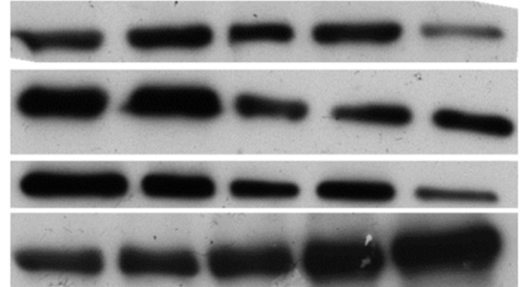

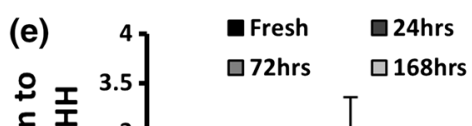

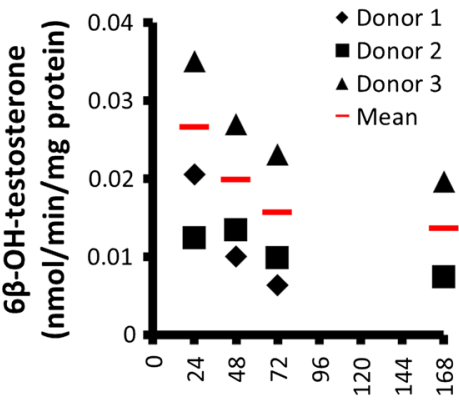

Time in culture (hrs)

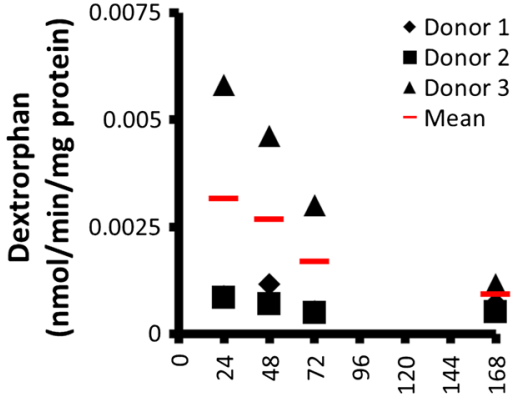

Time in culture (hrs)

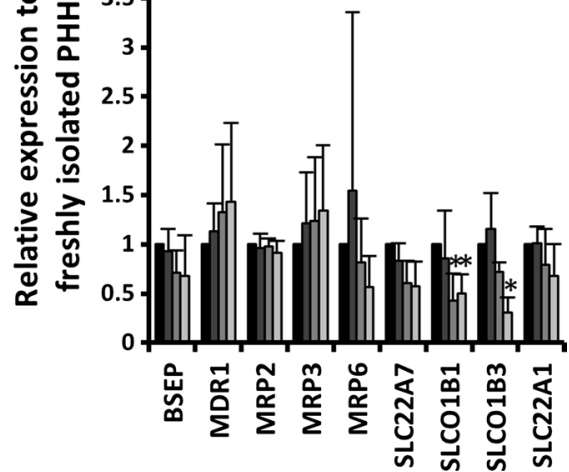


\Fig. 4 Loss of hepatic metabolic phenotype. a ADME phases I, II and III enzyme heatmap. Red up-regulated, green down-regulated. Intensity of colour relative fold change. Data derived from $n \geq 3$ donors unless stated $(\dagger) n=2$ or $(\ddagger) n=1$; b CYP450 s over $168 \mathrm{~h}$ $(n=5)$; error bars SD; one-way ANOVA: $(*) p<0.05$, (\#) $p<0.01)$; c western blots for CYP450 s and $\beta$-actin; $\mathbf{d}$ metabolic function of CYP3A (testosterone) and CYP2D6 (dextromethorphan) and detection of respective metabolites $(6 \beta-\mathrm{OH}$-testosterone and dextrorphan) detected by LC-MS-MS ( $n=3$; error bars SD); e ADME transporters protein expression during dedifferentiation. Error bars SD, ${ }^{*}$ significant $p<0.05$ by one-way ANOVA

dedifferentiation profiles (Fig. 4c). Conversely, $\beta$-actin was up-regulated during the culture period, which was in keeping with the majority of cytoskeletal proteins detected by iTRAQ proteomics (n.b. iTRAQ relative quantification of $\beta$-actin is not included in table $\mathrm{S} 2$ as it did not meet the stringent criteria used for the analysis) and the pathway analysis (Fig. 2a). Furthermore, analysis of CYP450 activity using probe substrates for CYP3A and 2D6 shows a loss of functional capacity that is mostly consistent with the loss of protein recorded by $\mathrm{iTRAQ}$ and western immunoblotting (Fig. 4d). Interestingly, despite the increase in CYP3A4 protein at $168 \mathrm{~h}$, no corresponding increase in CYP3A activity was detected; these results are in keeping with previous work which has shown that CYP protein expression does not correlate with activity in all situations (RodriguezAntona et al. 2002) and may represent the presence of the CYP3A4 protein in a non-functional state.

In contrast to the CYPs, the protein expression of the transporters identified as being important for drug influx and efflux in the liver (Zamek-Gliszczynski et al. 2012) is relatively stable throughout the 168-h culture. Only 2 of the 9 detected transporters are significantly down-regulated at $168 \mathrm{~h}$ ( $p<0.05$; Fig. 4e); interestingly, these are both influx transporters (SLCO1B1 and SLCO1B3). The general trend of the other detected drug influx transporters is also downwards, as are the trends for efflux transporters MRP6 and BSEP. Conversely, the remaining efflux transporters (MDR1, MRP2 and MRP3) show a maintenance or upward trend in expression during culture.

Comparison of the trend in the CYP and transporter proteins with previously published hepatocyte monolayer gene expression data (Richert et al. 2006) allows further investigation of the loss of metabolic competence (table S8). All CYPs with a reported gene expression $>30 \%$ at $72 \mathrm{~h}$ maintained protein expression $>60 \%$ at $168 \mathrm{~h}$. Conversely, at $72 \mathrm{~h}$, the mRNA of CYP1A2, 2A6, 2B6, 2C8, 2E1, 3A4, 4A11, 4F2, $4 \mathrm{~F} 3$ and $27 \mathrm{~A} 1$ is reported to drop to below $10 \%$ of freshly isolated PHH (Richert et al. 2006). When the protein expression of these CYPs is compared, the majority, with the exception of CYP4F2 and 4F3, show significant down-regulation at $168 \mathrm{~h}$, suggesting that the loss of CYPs can be mainly associated with reduced transcription. This association is shown in figure S4, demonstrating a good correlation between
mRNA at $72 \mathrm{~h}$ and protein at $168 \mathrm{~h}$ in the down-regulated CYPs. Some phase III proteins appear to show greater stability, including BSEP, SLC22A7 and SLC22A1 which have mRNA $<10 \%$ at $72 \mathrm{~h}$; however, despite a downward trend, they are not significantly down-regulated at $168 \mathrm{~h}$.

\section{Pathway analysis of ADME proteins reveals the pathways and transcription factors predicted to underlie the loss of specific metabolic functions}

Targeted analysis of the transcription factors that are associated with the ADME DEPs revealed the specific regulators, which are significantly perturbed and may cause the loss of ADME proteins during dedifferentiation (table S9). In keeping with published literature (Fraczek et al. 2013), HNF1 $\alpha, \mathrm{C} / \mathrm{EBP}$ and HNF1 $\beta$ were amongst the most significantly associated regulators with the loss of metabolic competence. Interestingly, FECH (ferrochelatase), a key enzyme in the production of heme, found to be significantly down-regulated at $168 \mathrm{~h}$ (table S2), is highly associated with the differentially expressed ADME proteins. SMARCB1 is also significantly associated with the ADME proteomic profile at $168 \mathrm{~h}$.

Investigation into the predicted transcription factor binding sites of differentially expressed and non-differentially expressed CYPs revealed the factors that may underlie the selectivity found in the loss of xenobiotic CYPs (Table S10). This analysis predicted binding sites for HSF2 to be highly enriched in the CYPs which were not differentially expressed, whereas ZEB1, FOXO1 and NF-Y binding sites are almost exclusively predicted to be located upstream of the differentially expressed CYPs.

\section{Discussion}

In this study, we have demonstrated the first use of global proteomics as a tool for investigating primary human hepatocyte dedifferentiation, identifying new targets and generating new hypotheses for future examination in simple and complex culture models.

Throughout the analysis, differentially expressed proteins and associated pathways were found at every time point, suggesting that these mechanisms are well conserved across donors. The number of significantly differentially expressed proteins increased at each time, which, together with the clustering analysis, suggests an increasing deviation from the freshly isolated donor phenotype. Grouping of these data showed that dedifferentiation is not solely a change in expression of whole protein classes, but that it is a complex, differentially controlled and active process, in which selected pathways and functions from many classes are up- or down-regulated. 
Cytochrome P450s has been strongly associated with a loss of expression during culture (Rodriguez-Antona et al. 2002), and this is further demonstrated here by the top three most down-regulated proteins at $168 \mathrm{~h}$ : CYP2A6, CYP2C8 and CYP4A11. However, this study allows for these changes to be put into the context of the global cell proteome, revealing the strikingly selective nature of dedifferentiation. Even within single P450 families, selectivity can be seen. For example, whilst CYP2A6 and CYP2C8 are the most down-regulated proteins, CYP2C18 and CYP2J2 remain unaltered by culture conditions. Of further interest, CYP2A6 has been reported to be the largest discriminant between foetal and adult hepatocytes (Rowe et al. 2013); here, we show CYP2A6 to be the largest discriminator between freshly isolated and $168 \mathrm{~h}$ cultured hepatocytes.

Understanding how this specificity works is a key question in determining new strategies for hepatocyte culture and for stem cell differentiation protocols. By comparing predicted transcription factor binding, we have been able to generate a list of enriched transcription factors associated with either differentially expressed or non-differentially expressed CYPs. Future investigations to elucidate the roles of these factors (e.g. HSF2, SP1 and ZEB1) may lead to a greater understanding of the selective loss/maintenance of metabolic competence during dedifferentiation. For example, ZEB1, a key mediator of epithelial-mesenchymal transition (EMT), may in part explain the link between the maintenance of epithelial phenotype and CYP expression.

Comparisons between differentially expressed and non-differentially expressed drug-metabolising proteins revealed no single factor which could mediate all the ADME-associated proteomic changes. The results corroborate previous reports showing the importance of HNF1 $\alpha$ alongside HNF1 $\beta$, C/EBP and FOXA1 in dedifferentiation (Padgham et al. 1993). Despite being a well-accepted major determinant of hepatic function (Odom et al. 2004), the lack of association of $\mathrm{HNF} 4 \alpha$ with differentially expressed ADME proteins is consistent with HNF4 $\alpha$ overexpression studies in rat hepatocytes, which failed to reestablish xenobiotic metabolic function (Naiki et al. 2005). The association of the enzyme ferrochelatase is also of interest. Ferrochelatase catalyses the final step of the heme production pathway, and thus, its loss of expression would likely reduce the intracellular heme concentration. CYPs are dependent on heme for function, and its loss during culture has been reported in mouse hepatocytes (Singh and Veltri 1991). HNF1 $\alpha$ has been shown to regulate ferrochelatase expression (Muppala et al. 2000) and may provide an additional indirect mechanism by which HNF1 $\alpha$ can alter ADME protein expression.

One potential cause of the change in the hepatocyte ADME and proteome-wide phenotype is through alterations in the accessibility of DNA to transcriptional machinery. Our results strongly predict a down-regulation of SMARCB1, a member of the BAF complex, which is thought to remodel chromatin structure (Wilson and Roberts 2011). SMARCB1 been shown to be essential for hepatocyte differentiation (Gresh et al. 2005), and recent work has also linked another member of the BAF complex, SMARCA4, to the enhancement of the hESC-derived HLC phenotype (Mobus et al. 2015). Alterations in chromatin accessibility and transcription factor binding are one of the key first steps in the reprogramming of somatic cells to induced pluripotent stem cells (Papp and Plath 2013), and similar fundamental mechanisms may play a role in the acquisition of the dedifferentiated phenotype. Efforts to intervene epigenetically using histone deacetylase and DNA methyltransferase inhibitors have been successful in maintaining expression of hepatic functions, although the exact mechanisms are unclear (Fraczek et al. 2012, 2013). Taken together, these results highlight the importance of epigenetic regulation in the dedifferentiation process; however, the consequences of these changes and the mechanisms which underlie them remain unclear and require further investigation.

Energy production is another fundamental cellular process affected by dedifferentiation that is highlighted by this study. The proteomic and functional data suggest a dramatic remodelling of the bioenergetic proteome during monolayer culture. These changes lead to a reduction in many mitochondrial-associated proteins, particularly those involved in lipid and fatty acid metabolism. Previous work has implicated AMPK signalling and mitochondrial fusion as part of the adaptation and repolarisation of hepatocytes in sandwich culture following isolation $(\mathrm{Fu}$ et al. 2013). Here we have additionally shown both AMPK and mitochondrial fusion pathways to be associated with the bioenergetic adaption to a monolayer culture system. Furthermore, given the remarkable stability of oxidative phosphorylation following plating, during a period of substantial phenotypic change, it appears that these essential processes are preferentially maintained, potentially at the expense of non-survival-essential proteins/functions. One possible driving factor of these findings is the MEK/ERK pathway, which is highlighted by this and other studies (Zellmer et al. 2010), as a factor in dedifferentiation and has previously been shown to reduce rotenone toxicity in neuronal cells (Jiang et al. 2006) and alter both mitochondrial function (Ripple et al. 2013) and lipid/fatty acid metabolism (Yousefi et al. 2012).

Our analysis revealed that the early stages of dedifferentiation are predominantly donor-dependent with the interindividual variation that exists in the starting donor phenotype being maintained throughout the first $72 \mathrm{~h}$ of culture. 
Hepatocytes are known to vary between donors (due to both genotypic and environmental factors) (Bhogal et al. 2011) and in function depending on their location/zone in the lobule portocentral axis (Allen et al. 2005); both of these factors also appear to influence the dedifferentiation profile during culture. Similarity to the isolated donor phenotype dissipates with time in culture in all cases, a convergence towards a reproducible dedifferentiated phenotype was observed. Taken together, these data suggest that the strictly defined culture system promotes a specific protein expression profile, and, as a consequence of the variable $\mathrm{PHH}$ phenotype at isolation, the proteome changes required to achieve this dedifferentiated phenotype are also variable. This information has profound implications for the use of hepatocytes for early drug discovery and prediction of DILI; the majority of toxicological endpoints are assessed within $72 \mathrm{~h}$ of isolation; thus, the variable dedifferentiation of hepatocytes demonstrated in this study is likely to have an impact on attempts to achieve consistent biological assessment of multiple chemical variables (e.g. libraries of new drugs).

Many of the earliest changes in protein expression during dedifferentiation are related to cell survival and the maintenance of homoeostatic functions. The up-regulation of stress-induced survival pathways may be considered as a double-edged sword, on the one hand preventing apoptosis/ necrosis and allowing cells to survive during culture, whilst on the other, playing an important role in the loss of the hepatic phenotype. Negative regulation of the hepatic phenotype has been reported to be true for the MEK/ERK and NFKB pathways (Elaut et al. 2006; Fraczek et al. 2013) and is predicted to be up-regulated by this study. Additionally, the most significantly predicted up-regulated factor at $168 \mathrm{~h}$ was heat shock factor 2 (HSF2). Overexpression studies have shown HSF2 to inhibit erythroid differentiation, but to our knowledge, HSF2 has not previously been linked to the hepatic differentiation status (Leppä et al. 1997). Such results emphasise the apparent trade-off between cell survival and a mature hepatic phenotype in traditional in vitro systems. Therefore, reducing cellular stress at all stages of culture, particularly during isolation, may be the most successful method of maintaining both cell viability and the hepatic phenotype.

Whilst some of the results yielded in this study may be specific to monolayer culture, the use of a rudimentary culture system has allowed the identification of a wide array of known and novel mechanisms through which dedifferentiation influences hepatic phenotype. We believe this approach enables a holistic understanding of the hepatocyte dedifferentiation process, and the knowledge gained in this study has the potential to enhance complex culture systems towards an improved physiological phenotype.
Open Access This article is distributed under the terms of the Creative Commons Attribution 4.0 International License (http://creativecommons.org/licenses/by/4.0/), which permits unrestricted use, distribution, and reproduction in any medium, provided you give appropriate credit to the original author(s) and the source, provide a link to the Creative Commons license, and indicate if changes were made.

\section{References}

Allen JW, Khetani SR, Bhatia SN (2005) In vitro zonation and toxicity in a hepatocyte bioreactor. Toxicol Sci 84(1):110-119. doi:10.1093/toxsci/kfi052

Bhogal RH, Hodson J, Bartlett DC et al (2011) Isolation of primary human hepatocytes from normal and diseased liver tissue: a one hundred liver experience. PLoS ONE 6(3):e18222. doi:10.1371/ journal.pone.0018222

Darnell M, Ulvestad M, Ellis E, Weidolf L, Andersson TB (2012) In vitro evaluation of major in vivo drug metabolic pathways using primary human hepatocytes and HepaRG cells in suspension and a dynamic three-dimensional bioreactor system. J Pharmacol Exp Ther 343(1):134-144

Elaut G, Henkens T, Papeleu P et al (2006) Molecular mechanisms underlying the dedifferentiation process of isolated hepatocytes and their cultures. Curr Drug Metab 7(6):629-660

Fraczek JE, Vinken M, Tourwe D, Vanhaecke T, Rogiers V (2012) Synergetic effects of DNA demethylation and histone deacetylase inhibition in primary rat hepatocytes. Invest New Drugs 30(4):1715-1724. doi:10.1007/s10637-011-9659-8

Fraczek J, Bolleyn J, Vanhaecke T, Rogiers V, Vinken M (2013) Primary hepatocyte cultures for pharmaco-toxicological studies: at the busy crossroad of various anti-dedifferentiation strategies. Arch Toxicol 87(4):577-610. doi:10.1007/s00204-012-0983-3

Fu D, Mitra K, Sengupta P, Jarnik M, Lippincott-Schwartz J, Arias IM (2013) Coordinated elevation of mitochondrial oxidative phosphorylation and autophagy help drive hepatocyte polarization. Proc Natl Acad Sci 110(18):7288-7293. doi:10.1073/ pnas. 1304285110

Godoy P, Hengstler JG, Ilkavets I et al (2009) Extracellular matrix modulates sensitivity of hepatocytes to fibroblastoid dedifferentiation and transforming growth factor beta-induced apoptosis. Hepatology 49(6):2031-2043

Godoy P, Hewitt NJ, Albrecht U et al (2013) Recent advances in 2D and $3 \mathrm{D}$ in vitro systems using primary hepatocytes, alternative hepatocyte sources and non-parenchymal liver cells and their use in investigating mechanisms of hepatotoxicity, cell signaling and ADME. Arch Toxicol 87(8):1315-1530

Gresh L, Bourachot B, Reimann A et al (2005) The SWI/SNF chromatin-remodeling complex subunit SNF5 is essential for hepatocyte differentiation. EMBO J 24(18):3313-3324. doi:10.1038/ sj.emboj. 7600802

Guo L, Dial S, Shi L et al (2011) Similarities and differences in the expression of drug-metabolizing enzymes between human hepatic cell lines and primary human hepatocytes. Drug Metab Dispos 39(3):528-538. doi:10.1124/dmd.110.035873

Jiang Q, Yan Z, Feng J (2006) Neurotrophic factors stabilize microtubules and protect against rotenone toxicity on dopaminergic neurons. J Biol Chem 281(39):29391-29400. doi:10.1074/jbc. M602740200

Jungermann K, Kietzmann T (1996) Zonation of parenchymal and nonparenchymal metabolism in liver. Annu Rev Nutr 16:179203. doi:10.1146/annurev.nu.16.070196.001143 
Kia R, Sison RL, Heslop J et al (2013) Stem cell-derived hepatocytes as a predictive model for drug-induced liver injury: are we there yet? Br J Clin Pharmacol 75(4):885-896

Kim Y, Lasher CD, Milford LM, Murali TM, Rajagopalan P (2010) A comparative study of genome-wide transcriptional profiles of primary hepatocytes in collagen sandwich and monolayer cultures. Tissue Eng Part C Methods 16(6):1449-1460

Lasher CD, Rajagopalan P, Murali TM (2011) Discovering networks of perturbed biological processes in hepatocyte cultures. PLoS ONE 6(1):0015247

LeCluyse EL, Alexandre E, Hamilton GA et al (2005) Isolation and culture of primary human hepatocytes. Methods Mol Biol 290:207-229

Leppä S, Pirkkala L, Saarento H, Sarge KD, Sistonen L (1997) Overexpression of HSF2- $\beta$ inhibits hemin-induced heat shock gene expression and erythroid differentiation in K562 cells. J Biol Chem 272(24):15293-15298. doi:10.1074/jbc.272.24.15293

Marinescu VD, Kohane IS, Riva A (2005) The MAPPER database: a multi-genome catalog of putative transcription factor binding sites. Nucleic Acids Res 33(Suppl 1):D91-D97. doi:10.1093/nar/ gki103

Mi H, Muruganujan A, Casagrande JT, Thomas PD (2013) Largescale gene function analysis with the PANTHER classification system. Nat Protoc 8(8):1551-1566

Mobus S, Yang D, Yuan Q et al (2015) MicroRNA-199a-5p inhibition enhances the liver repopulation ability of human embryonic stem cell-derived hepatic cells. J Hepatol 62(1):101-110. doi:10.1016/j.jhep.2014.08.016

Muppala V, Lin C-S, Lee Y-H (2000) The role of HNF-1 $\alpha$ in controlling hepatic catalase activity. Mol Pharmacol 57(1):93-100

Naiki T, Nagaki M, Asano T, Kimata T, Moriwaki H (2005) Adenovirus-mediated hepatocyte nuclear factor-4alpha overexpression maintains liver phenotype in cultured rat hepatocytes. Biochem Biophys Res Commun 335(2):496-500. doi:10.1016/j. bbrc.2005.07.102

No DY, Lee S-A, Choi YY et al (2012) Functional 3D human primary hepatocyte spheroids made by co-culturing hepatocytes from partial hepatectomy specimens and human adipose-derived stem cells. PLoS ONE 7(12):e50723. doi:10.1371/journal. pone. 0050723

Odom DT, Zizlsperger N, Gordon DB et al (2004) Control of pancreas and liver gene expression by HNF transcription factors. Science 303(5662):1378-1381. doi:10.1126/science.1089769

Padgham CR, Boyle CC, Wang XJ, Raleigh SM, Wright MC, Paine AJ (1993) Alteration of transcription factor mRNAs during the isolation and culture of rat hepatocytes suggests the activation of a proliferative mode underlies their de-differentiation. Biochem Biophys Res Commun 197(2):599-605. doi:10.1006/ bbrc. 1993.2521

Papp B, Plath K (2013) Epigenetics of reprogramming to induced pluripotency. Cell 152(6):1324-1343. doi:10.1016/j. cell.2013.02.043

Rambhatla L, Chiu CP, Kundu P, Peng Y, Carpenter MK (2003) Generation of hepatocyte-like cells from human embryonic stem cells. Cell Transpl 12(1):1-11

Richert L, Liguori MJ, Abadie C et al (2006) Gene expression in human hepatocytes in suspension after isolation is similar to the liver of origin, is not affected by hepatocyte cold storage and cryopreservation, but is strongly changed after hepatocyte plating. Drug Metab Dispos 34(5):870-879. doi:10.1124/ dmd.105.007708

Ripple MO, Kim N, Springett R (2013) Acute mitochondrial inhibition by mitogen-activated protein kinase/extracellular signal-regulated kinase kinase (MEK) 1/2 inhibitors regulates proliferation. J Biol Chem 288(5):2933-2940. doi:10.1074/jbc.M112.430082

Ritchie ME, Phipson B, Wu D et al (2015) limma powers differential expression analyses for RNA-sequencing and microarray studies. Nucleic Acids Res 43(7):e47. doi:10.1093/nar/gkv007

Rodriguez-Antona C, Donato MT, Boobis A et al (2002) Cytochrome $\mathrm{P} 450$ expression in human hepatocytes and hepatoma cell lines: molecular mechanisms that determine lower expression in cultured cells. Xenobiotica 32(6):505-520. doi:10.1080/00498250210128675

Rowe C, Goldring CE, Kitteringham NR et al (2010) Network analysis of primary hepatocyte dedifferentiation using a shotgun proteomics approach. J Proteome Res 9(5):2658-2668

Rowe C, Gerrard DT, Jenkins R et al (2013) Proteome-wide analyses of human hepatocytes during differentiation and dedifferentiation. Hepatology 58(2):799-809. doi:10.1002/hep.26414

Schwanhausser B, Busse D, Li N et al (2011) Global quantification of mammalian gene expression control. Nature 473(7347):337-342

Shan J, Schwartz RE, Ross NT et al (2013) Identification of small molecules for human hepatocyte expansion and iPS differentiation. Nat Chem Biol 9(8):514-520

Singh G, Veltri KL (1991) A mechanism for the loss of cytochrome $\mathrm{P}-450$ in primary mouse hepatocytes. Mol Cell Biochem 108(2):151-156

Sistonen J, Fuselli S, Palo JU, Chauhan N, Padh H, Sajantila A (2009) Pharmacogenetic variation at CYP2C9, CYP2C19, and CYP2D6 at global and microgeographic scales. Pharmacogenet Genomics 19(2):170-179. doi:10.1097/FPC.0b013e32831ebb30

Thomas S, Bonchev D (2010) A survey of current software for network analysis in molecular biology. Hum Genomics 4(5):353-360

Tostoes RM, Leite SB, Serra M et al (2012) Human liver cell spheroids in extended perfusion bioreactor culture for repeated-dose drug testing. Hepatology 55(4):1227-1236

Wilson BG, Roberts CWM (2011) SWI/SNF nucleosome remodellers and cancer. Nat Rev Cancer 11(7):481-492

Yousefi B, Darabi M, Baradaran B et al (2012) Inhibition of MEK/ ERK1/2 signaling affects the fatty acid composition of HepG2 human hepatic cell line. BioImpacts: BI 2(3):145-150. doi:10.5681/bi.2012.019

Zamek-Gliszczynski MJ, Hoffmaster KA, Tweedie DJ, Giacomini KM, Hillgren KM (2012) Highlights from the international transporter consortium second workshop. Clin Pharmacol Ther 92(5):553-556. doi:10.1038/clpt.2012.126

Zellmer S, Schmidt-Heck W, Godoy P et al (2010) Transcription factors ETF, E2F, and SP-1 are involved in cytokineindependent proliferation of murine hepatocytes. Hepatology 52(6):2127-2136 